\title{
*-Valuations and Hermitian Forms on Skew Fields
}

\author{
Thomas C. Craven \\ Department of Mathematics \\ University of Hawaii, Honolulu \\ Hawaii 96822
}

\begin{abstract}
This paper is a survey of the literature on ways in which the concept of ordering can be extended to the setting of a division ring with involution and the main results for these extensions.
\end{abstract}

\section{Introduction and notation}

This paper updates the survey paper of Craven [13] written several years ago. It is more focused on $*$-fields which have an ordering of some sort, with some of the more peripheral references of the earlier paper removed and many new references and results added to reflect the recent interest in the area. No proofs are given, but an effort is made to refer the reader to all relevant sources.

Let $D$ be a skew field (division ring) with an involution $*$ (an anti-automorphism of order 2$)$. We shall refer to $(D, *)$ as a $*$-field. Most of the topics of this paper are generalizations of results in the theory of formally real fields, which have been very nicely presented by T. Y. Lam [27]. Indeed, if the involution $*$ is the identity, then $D$ is necessarily commutative, so we have a direct generalization. When $D$ is noncommutative, the involution is necessary in order to have a generalization of the role of the algebraic theory of quadratic forms in the theory of ordered fields. In Section 2, we shall introduce all of the various types of orderings which have been proposed for $*$-fields. Section 3 looks at how well these various definitions work with valuation theory. In Section 4 the relationship with hermitian forms will be explored. Finally, in Section 5 we shall look at the recent extension of this theory to rings and, in particular, to $\mathrm{C}^{*}$-algebras.

Examples of $*$-fields As with commutative fields, in order to have an ordering of any sort, we limit ourselves to characteristic zero. When $*$ is not the identity, commutative examples have the form of a field extension $K / F$ of degree 2 , where the involution is the nontrivial automorphism generating the Galois group. The simplest noncommutative examples are quaternion algebras, generated, say, by $i, j$ over a field $F$, with involution given by $i^{*}=-i, j^{*}=-j$; we refer to such algebras as standard quaternion algebras. These examples play a rather trivial role in the

2000 Mathematics Subject Classification. Primary 11E39, 11E81, 12D15, 13J30; Secondary 12J15, 12J25, 16K99, 16W10. 
theory as most results follow readily from a knowledge of the theory of formally real fields.

More interesting examples which have been constructed with orderings of various types include nonstandard quaternion algebras (a nonstandard involution causes much different behavior), tensor products of such algebras, crossed products, and skew fields of polynomials or Laurent series. More recently, Morandi and Sethuramen $[36,37]$ have constructed indecomposable and noncrossed product division algebras with Baer orderings.

Notation We write $S(D)=\left\{d \in D \mid d^{*}=d\right\}$ for the set of symmetric (self-adjoint) elements of $(D, *)$ and denote the center of $D$ by $Z_{D}$. For any subset $A \subseteq D$, we shall use the notation $A^{\times}$to denote the set of nonzero elements in $A$. We write multiplicative commutators in the form $[a, b]=a b a^{-1} b^{-1}$, and, more generally, for subsets $A, B \subseteq D^{\times}$, we write $[A, B]=\{[a, b] \mid a \in A, b \in B\}$. A basic idea behind orderings in this context is that all norms (elements of the form $d d^{*}$ for $d \in D^{\times}$) should be positive. These replace squares in the formally real case. In some situations, certain commutators must also be positive, leading us to define $\Sigma(D)$ to be the set of all sums of products of norms and elements of $\left[D^{\times}, S(D)^{\times}\right]$.

Structure theory for $*$-fields We shall see that some types of orderings only exist in skew fields which are purely transcendental over their centers, while others exist in division rings finite-dimensional over their centers. There is very little known structure theory in the former case. We mention two general facts. One is that either $Z_{D} \subseteq S(D)$ (in which case, the involution is said to be of the first kind) or the center is a quadratic extension of the field of central symmetric elements (in which case, the involution is said to be of the second kind). The second fact is a result due to Dieudonné [19, Lemma 1]: if $(D, *)$ is noncommutative and not a standard quaternion algebra, then the symmetric elements generate $D$ as a ring. This gives a strong indication of why the commutative and standard quaternion division algebra cases are special.

The existence of certain types of orderings puts strong conditions on finitedimensional $*$-fields. Some of these results can be found in Chacron and Wadsworth [7] (see $\S 3$ below) and Idris [25] (see $\S 2$ below).

\section{Orderings}

In this section we shall describe the many different definitions of ordering and semiordering which have been proposed for a $*$-field. If the involution is not the identity, no definition can give a total ordering of $D$ since skew elements (i.e., $\left.d^{*}=-d\right)$ can be neither positive nor negative. All definitions give a total ordering of the symmetric elements $S(D)$. The definitions vary in whether they are restricted to $S(D)$ or are defined on larger subsets of $D$. More importantly, they vary in the form of their multiplicative properties. Recall that in a formally real field, a semiordering differs from an ordering in requiring closure only under multiplication by squares. See Prestel [39, p. 5].

All orderings will be defined in terms of their positive cone, a subset $P \subset D$, with the understanding that $a<b$ in $D$ is equivalent to $b-a \in P$. Most definitions come in pairs, one for an ordering with $P \subset S(D)$, and the other an "extended" version where some nonsymmetric elements are ordered as well. The relationship between these versions is not always apparent. The most important role of the extended versions has usually been to aid in proofs. All definitions are designed so 
that the set $P=\mathbb{R}^{+}$of positive real numbers works as an ordering for each of $\mathbb{R}, \mathbb{C}$ (complex numbers with conjugation as the involution) and $\mathbb{H}$ (real quaternions with the standard involution).

The subject began with a definition given by Baer [2, Chap. IV, App. I]. This definition has the advantages that it works well with hermitian forms (see $\S 4$ ) and has examples among finite-dimensional as well as infinite-dimensional division algebras. We begin with definitions which define a semiordering in the case where $D$ is commutative and $*$ is the identity.

Definition 2.1 A Baer ordering on $(D, *)$ is a subset $P \subseteq S(D)^{\times}$satisfying $P+P \subseteq P, d P d^{*} \subseteq P$ for all $d \in D^{\times}, 1 \in P$, and $P \cup-P=S(D)^{\times}$.

As noted in Chacron [3], the same definition can be used without restricting $P$ to be a subset of $S(D)$. When needed, we shall call this an extended Baer ordering. An alternative to this definition of ordering was suggested by Chacron [3], the difference being in how the norm $d d^{*}$ is used in the multiplicative requirement. It was initially proposed in the following extended version.

Definition 2.2 A c-ordering on $(D, *)$ is a $*$-closed subset $P \subseteq D^{\times}$satisfying $P+P \subseteq P, P d d^{*} \subseteq P$ for all $d \in D^{\times}, 1 \in P$, and $P \cup-P \supseteq S(D)^{\times}$.

The following strengthening of the axioms for a c-ordering was proposed in Chacron [5, p.17] to make the valuation theory work better (see $\S 3$ ).

Definition 2.3 A normal c-ordering is a c-ordering $P$ with the property that, for any element $u \in S(D)$ which is bounded above and below by positive rational numbers, one has sus $\in P$ for all $s \in S(D)^{\times}$.

Chacron [6] has also introduced the restriction of a c-ordering to the symmetric elements. A formal definition can be given by

Definition 2.4 A symmetric c-ordering is a subset $P \subset S(D)^{\times}$satisfying $1 \in P, P+P \subseteq P, P \cup-P=S(D)^{\times}$and, for all $p \in P$ and $x$ a product of norms, $p x+x^{*} p \in P$.

Chacron actually gave a slightly less restrictive definition by only assuming that the $x$ in the definition was a norm. However, the intersection of a c-ordering with the symmetric elements actually satisfies the stronger condition of this definition, and this form makes it possible to use valuation theory to show that any symmetric c-ordering has an extension to a c-ordering (see Craven [14]). It is not known if the two definitions are equivalent.

Holland [22] was the first to notice that it is quite reasonable to require closure under multiplication also, thus introducing the first true generalization of orderings (which he called strong orderings). These satisfy the axioms of both Baer orderings and c-orderings. One disadvantage of these orderings is that, just as with total orderings of a skew field without involution, such orderings can exist in a noncommutative skew field $D$ only when $D$ is purely transcendental over its center or $D$ is a standard quaternion algebra.

Definition 2.5 An extended $*$-ordering on $(D, *)$ is a $*$-closed subset $P \subseteq D^{\times}$ satisfying $P+P \subseteq P, d P d^{*} \subseteq P$ for all $d \in D^{\times}, 1 \in P, P \cup-P \supseteq S(D)^{\times}$, and $P \cdot P \subseteq P$. 
Note that an extended $*$-ordering $P$ is a normal subgroup of $D^{\times}$; indeed, if $x, y \in P$, then so are $x^{-1}=x^{*}\left(x^{-1 *} x^{-1}\right)$ and $y x y^{-1}=\left(y x y^{*}\right)\left(y y^{*}\right)^{-1}$. The restricted version of this was introduced by Idris [24], who called it a Jordan ordering. It is simply a Baer ordering closed under a symmetric version of multiplication.

Definition 2.6 $\mathrm{A} *$-ordering on $(D, *)$ is a Baer ordering $P$ satisfying $x y+y x \in$ $P$ for all $x, y \in P$.

The next two definitions were introduced by Craven [11]; they give a version of semiordering which works well with $*$-orderings, in that virtually all results concerning semiorderings in the commutative theory can be extended to these. Again, their existence implies that $D$ is infinite-dimensional over its center. As in the commutative theory, it is also possible to make the definitions relative to a preordering (cf. [11, §3]) in place of $\Sigma(D)$.

Definition 2.7 A *-semiordering on $(D, *)$ is Baer ordering $P$ satisfying $x y+$ $y^{*} x \in P$ for all $x \in P$ and $y \in \Sigma(D)$.

Using $\prod S(D)$ to denote the set of all nonzero products of symmetric elements, we can state

Definition 2.8 An extended $*$-semiordering on $(D, *)$ is an extended Baer ordering $P$ satisfying $P \cup-P \supseteq \prod S(D)$ and $P \cdot \Sigma(D) \subseteq P$.

The final definition to be considered here was introduced by Leung [28]. It has stronger multiplicative properties than a Baer ordering, but still can exist in the case of division rings finite-dimensional over their centers. For this finite-dimensional situation, the orderings are determined by their restriction to the center, so the commutative theory extends more easily. In the infinite-dimensional case, little is known.

Definition 2.9 A weak $*$-ordering on $(D, *)$ is a Baer ordering $P$ satisfying $a P=P$ for any $a \in P \cap Z_{D}$.

Archimedean orderings The earliest work with these orderings gives characterizations of the archimedean ones. Holland [19, Theorem 2] shows that every archimedean Baer ordered $*$-field is $*$ and order isomorphic to a subfield of the real numbers, complex numbers or real quaternions. The same holds true for a c-ordered field since archimedean c-orderings are, in fact, Baer orderings (cf. Chacron [3]).

Artin-Schreier theory The most fundamental questions to ask are those concerning the extension of the theorems of Artin and Schreier on the existence of orderings and on intersections of orderings. Note that sums of nonzero norms are positive in all types of orderings. However, to obtain the existence of an ordering (any type), it is not sufficient to require that zero cannot be written as a sum of nonzero norms (cf. Holland [19, Corollary 5]). An alternative is to require that $\sum d_{i} s d_{i}^{*} \neq 0$ whenever $s \in S(D)^{\times}$and $d_{i} \in D^{\times}$. It remains an open question as to whether this condition is sufficient to force $D$ to even have a Baer ordering. Leung [29, Theorem 2.9] [30, Theorem 3.4] is able to get results which formally resemble Artin-Schreier theory, but the defined objects are not sufficiently computable to resolve this question.

For c-orderings, the situation is somewhat better. If zero cannot be written as a sum of products of nonzero norms, then $D$ has a c-ordering by the usual Zorn's lemma argument. See Chacron [3, Theorem 2]. 
For $*$-orderings and extended $*$-orderings, the usual Artin-Schreier results still hold (cf. Handelman [18] in the commutative case and Holland [22] for extended $*$ orderings). The intersection of all extended $*$-orderings is $\Sigma(D)$ and the intersection of all $*$-orderings is $\Sigma(D) \cap S(D)$. Idris [24] has shown (see $\S 3$ for details) that every $*$-ordering extends uniquely to a maximal extended $*$-ordering, so it is easy to correlate results between the two types of orderings.

Topology Just as for orderings of commutative fields, one can topologize the sets of Baer orderings, *-orderings, c-orderings, etc. This has been done for Baer orderings in Craven [9] and Leung [29], for $*$-orderings in Craven [9], [11], [12], for $*$-semiorderings in [9], for weak $*$-orderings in [29] and for c-orderings in McDonough [35]. In all of these cases, the topology is generated by the subbasis consisting of all sets $H(a)$, defined to be the set of all orderings in which $a \in S(D)^{\times}$ is positive. As in the commutative case, the spaces are compact, Hausdorff and totally disconnected. In the case of $*$-orderings, this topology can also be obtained as the Zariski topology on $W R(D, *)$, a ring of equivalence classes of hermitian forms defined in Craven [11] (see $\S 4$ for more information). In the sequel, we shall write $X_{D}$ for the topological space of all *-orderings on $(D, *)$ and $Y_{D}$ for the space of all Baer orderings.

One of the earliest questions considered (in the commutative theory) regarding this topology was to ask when every clopen (both closed and open) subset can be realized as a set of the form $H(a)$ for some $a \in S(D)$ (cf. [26, Theorem 3.20], $[39, \S 9],[27, \S 17])$. This is known as the strong approximation property (SAP). Most of the many equivalent conditions for commutative fields can be generalized. For example, the space of $*$-orderings $X_{D}$ satisfies SAP if and only if every *semiordering is actually a $*$-ordering [9, Theorem 4.1]. And, for the case of $D$ being finite-dimensional over its center, the space of Baer orderings satisfies SAP if and only if every Baer ordering is actually a weak *-ordering; furthermore, in this case, $D$ either is commutative, a standard quaternion algebra, or $\left[D: Z_{D}\right]$ is odd (see Leung $[29, \S 5]$ ).

We introduce "fans" here primarily because they will be needed in $\S 4$. However, they are also related to the strong approximation property. We shall make the definitions in the $*$-ordering situation. A preordering is a subset $T \subseteq S(D)^{\times}$satisfying $T+T \subseteq T, d T d^{*} \subseteq T$ for all $d \in D^{\times}, S(D) \cap \Sigma(D) \subseteq T, 1 \in T$ and $x y+y^{*} x^{*} \in T$ whenever $x+x^{*}, y+y^{*} \in T$ for $x, y$ in the subgroup $S(D)^{\times} \cdot \Sigma(D)$ of $D^{\times}$(see Craven [11, §3]). A preordering is called a fan if, for all $a \in S(D)^{\times}$, if $a \notin-T$, then $1+a \in T \cup a T$ (see [10, Theorem 2.8]). For any preordering $T$, we write $X_{T}=\left\{P \in X_{D} \mid P \supseteq T\right\}$. Then SAP holds if and only if $\left|X_{T}\right| \leq 2$ for all fans $T$. Indeed, if $\left|X_{T}\right| \geq 4$, then there exist four $*$-orderings in $X_{T}$ such that three of them cannot be separated from the fourth by any element $a \in S(D)$. Conversely, if such separation is always possible, then one can show that SAP holds (see Theorem 4.3 below). A similar result holds for Baer orderings when $D$ is finite-dimensional. See Leung [29, Theorem 5.6].

\section{Valuations and orderings}

For commutative fields, every ordering or semiordering has an associated valuation ring; in fact, it has one for which the residue class field has an induced archimedean ordering or semiordering. Noncommutativity creates problems which are, in some respects, partially compensated for by the involution. 
Let $P$ be a Baer ordering of $(D, *)$. To $P$ we associate the set $A(P)=\{d \in$ $D \mid n-d d^{*} \in P$ for some $\left.n \in \mathbb{Z}\right\}$. This was first studied by Holland [20], where he shows that $A(P)$ is a total subring of $D$ (i.e., $d \in A(P)$ or $d^{-1} \in A(P)$ for every nonzero $d \in D$ ), with unique (two-sided) maximal ideal of infinitesimal elements $\mathfrak{m}_{P}=\left\{d \in A(P) \mid q-d d^{*} \in P\right.$ for all $\left.q \in \mathbb{Q}^{+}\right\}$. To be a valuation ring in the sense of Schilling [41], the ring $A(P)$ must also be invariant under inner automorphisms of $D$. All the early examples of Baer orderings were, in fact, invariant leaving the existence of noninvariant ones open for several years. Morandi and Wadsworth [38] have constructed examples in every possible finite dimension (of $D$ over its center) of Baer ordered $*$-fields for which $A(P)$ is not invariant. In any case, the residue (skew) field has an induced involution and an induced archimedean Baer ordering, making it isomorphic to a sub- $*$-field of the real quaternions $\mathbb{H}$. Holland $[20, \S 4]$ showed that there is an associated order valuation $v$, but that the set of values $\Gamma_{v}$ is not generally a group unless $v$ is a $*$-valuation (i.e. $v(d)=v\left(d^{*}\right)$ for all $\left.d \in D\right)$. In this case it is necessarily an abelian group since $v(a)+v(b)=v(a b)=v\left(b^{*} a^{*}\right)=v(b)+v(a)$.

Compatibility We shall follow Leung [28] and Craven [11, §4] in defining compatibility here. We say a $*$-valuation $v$ and a Baer ordering $P$ are compatible if the valuation ring $A_{v}$ is convex with respect to $P$, and are fully compatible if $0<a \leq b$ with respect to $P$ implies $v(a) \geq v(b)$ in $\Gamma_{v}$. The latter, stronger definition is the one often used for compatibility in the literature. It necessary to make a distinction since any Baer ordering is compatible with its order valuation, but may not be fully compatible with any valuation, even in the commutative case with identity involution (cf. Prestel [39, Theorem 7.17]). For $*$-orderings, the conditions are equivalent (cf. Craven [11, Theorem 4.2]), as they are for ordinary orderings of a commutative field.

Lifting theorems For a $*$-valuation $v$, lifting Baer orderings from the residue field back to $D$ encounters the following complication (see Holland [20], Craven [8]). For any nonzero symmetric element $s \in S(D)$, there is an involution \# induced on the residue field $D_{v}$ defined by $y^{\#}=\overline{s x s^{-1}}$, where $x \in D, \bar{x}=y \in D_{v}$. The element $s$ is called smooth if the induced involution \# is conjugate to $*$ (induced on $D_{v}$ by the involution $*$ on $\left.D\right)$. The valuation is called smooth if each equivalence class $v^{-1}(\gamma), \gamma \in \Gamma_{v}$, which contains some symmetric element, contains a smooth symmetric element. For a smooth valuation $v$, one can completely characterize the set of Baer orderings of $D$ fully compatible with $v$ by generalizing the usual theorems lifting orderings from the residue field. See [39, Theorem 7.8] for the case of semiorderings of commutative fields with identity involution and $[8$, Theorem $3.4]$ for the general case. A simpler situation occurs when $D$ is of odd dimension over its center (see Leung [29, Prop. 3.5, 3.6]).

With a slightly stronger version of smoothness to handle products (cf. Craven $[8$, Definition 2.1]), this characterization can also be carried out for $*$-orderings $[8$, Theorem 4.3]. To obtain a theorem which is easier to state, we consider a stronger condition on the valuation; we say a $*$-valuation is real if the commutator subgroup $\left[D^{\times}, S(D)^{\times}\right]$is mapped into some extended $*$-ordering of the residue field. This is equivalent to requiring that all $*$-orderings lift (cf. Craven [9, Proposition 2.2]), or that $\left[D^{\times}, S(D)^{\times}\right]$maps into $\Sigma\left(D_{v}\right)$ (cf. Craven [10, Proposition 2.1]). In particular, the order valuation of a $*$-ordering is always real (cf. Holland $[22, \S 5]$ ). 
Theorem 3.1 Craven [10, Theorem 2.2] Let $v$ be a real $*$-valuation on $(D, *)$. Let $S\left(\Gamma_{v}\right)=\left\{\gamma \in \Gamma_{v} \mid \gamma=v(s)\right.$ for some $\left.s \in S(D)\right\}$. Then there is a homeomorphism of $X_{D}^{v}$, the subspace of $X_{D}$ consisting of $*$-orderings fully compatible with $v$, with $X_{D_{v}} \times \operatorname{hom}(S(\Gamma) / 2 \Gamma,\{ \pm 1\})$, where $\operatorname{hom}(S(\Gamma) / 2 \Gamma,\{ \pm 1\})$ is given the character group topology.

Extension questions For both $*$-orderings and c-orderings, a natural question to ask is which extended ones intersect with $S(D)$ to give a particular restricted one. Much has been learned about this in the past few years, and for $*$-orderings valuation theory plays a fundamental role. A result of Idris establishes a connection between $*$-orderings and their maximal extensions to extended $*$-orderings. This connection is extremely valuable for its use in proving theorems about these orderings. See also Craven [8, Theorem 2.5] for a generalization to arbitrary $*$-valuations.

Theorem 3.2 Idris [24, Chapter 2, §3] Let $P$ be a *-ordering with order valuation v. Set $Q=\left\{s+k \mid s \in P, k^{*}=-k, v(k)>v(s)\right\}$. Then $Q$ is a maximal extended $*$-ordering with order valuation $v$ and satisfying $Q \cap S(D)=P$.

The following example shows that there may be infinitely many extensions of a given $*$-ordering, even when $D$ is commutative (where extended $*$-orderings are, in fact, c-orderings).

Example Let $K=\mathbb{R}((x))$ be the field of formal Laurent series over the real numbers and let $F$ be the subfield $\mathbb{R}\left(\left(x^{2}\right)\right)$. Let $*$ be the nontrivial automorphism of $K$ over $F$ (i.e. $f(x)^{*}=f(-x)$ ). Note that $S(K)=F$. As is well known, the field $F$ has exactly two (ordinary) orderings. Let $P \subseteq F$ be the ordering of $F$ in which $x^{2}$ is negative; that is, $P=\left\{\sum_{i=-n}^{\infty} a_{i} x^{2 i} \mid(-1)^{n} a_{-n}>0\right\}$. Since, for $f(x)=$ $\sum_{i=-n}^{\infty} a_{i} x^{i} \in K$, we have $f(x) f(x)^{*}=f(x) f(-x)=(-1)^{n} a_{-n}^{2} x^{-2 n}+\cdots \in P$, the set $P$ is a $*$-ordering of $K$. The largest extended $*$-ordering containing $P$ is the set $P_{\max }=\left\{\sum_{i=-n}^{\infty} a_{i} x^{i} \in K \mid n\right.$ is even and $\left.(-1)^{n / 2} a_{-n}>0\right\}$, obtained from Theorem 3.2. It is not difficult to check that there are infinitely many extended *-orderings between $P$ and $P_{\max }$, namely, all sets $P_{t}$, where $t$ is an odd integer and $P_{t}$ consists of all finite sums of elements from $\bigcup_{n=0}^{\infty}\left(1 \pm x^{t}\right)^{n} P$.

It turns out that this example shows the full story for $*$-orderings. Let $\Gamma_{v}^{+}=$ $\left\{v(k) \in \Gamma_{v} \mid k \in D^{\times}, v(k)>0, k^{*}=-k\right\} \cup\{v(0)=\infty\}$. In our example above, $\Gamma_{v}^{+}$consists of the positive odd integers and infinity.

Theorem 3.3 Craven [14, Theorem 2.3], corrected here Let $D$ be a $*$-field with $a *$-ordering $P$ and let $v$ be the associated order valuation with value group $\Gamma_{v}$. Then there is a one-to-one correspondence between extended $*$-orderings $Q$ containing $P$ and convex subsets $A \subseteq \Gamma_{v}^{+}$containing $\left\{v\left(s_{1} s_{2} s_{1}^{-1} s_{2}^{-1}-1\right) \mid s_{1}, s_{2} \in S(D)^{\times}\right\}$ defined by $Q_{A}=\left\{s+k \mid s \in P, k^{*}=-k, v(k)-v(s) \in A\right\}$ and $A_{Q}=\{v(k) \mid$ $1+k \in Q\}$.

For the orderings which are not multiplicative (c-orderings, Baer orderings), the restrictions to the symmetric elements can again be extended as in this theorem as long as $v$ is compatible with the ordering. But the converse no longer holds as there are now typically many more extensions. For Baer orderings, if we denote the set of skew elements by $S k(D)$, they are given by

Proposition 3.4 Craven [14, Proposition 3.3] Let $P$ be a Baer ordering of $(D, *)$. Let $B$ be any subset of $P+S k(D)$ such that (1) $B+B \subseteq B$, (2) $d B d^{*} \subseteq B$ 
for any $d \in D^{\times}$, (3) $B$ is $*$-closed and (4) $P \subseteq B$. Then $B$ is an extended Baer ordering and every extended Baer ordering arises in this way.

c-orderings Chacron [5] defines a c-valuation to be a $*$-valuation $w$ with a slightly weaker condition for reality (reflecting the fact that c-orderings are not necessarily closed under products); to be precise, $w(a+b)=\min (w(a), w(b))$ for $a$ and $b$ sums of norms and $d a a^{*} d^{-1}\left(a a^{*}\right)^{-1}$ maps to 1 in the residue field for all $a, d \in D^{\times}$. Order valuations behave much the same as with Baer orderings: the associated ring is total, but not necessarily invariant. Even if it is invariant, the valuation may not be a c-valuation [5]. One of the major results for c-orderings is a characterization of those c-orderings for which the order valuation is a c-valuation.

Theorem 3.5 Chacron [5, Theorem 3.1.9] Let $P$ be a c-ordering of $(D, *)$. The associated order valuation is a c-valuation if and only if $P$ is a normal c-ordering.

There are also some important facts known about the structure of finitedimensional c-valued division rings.

Theorem 3.6 Chacron and Wadsworth [7, Theorem 2.1] Let D be any finitedimensional noncommutative division ring with c-valuation $v$ and associated maximal ideal $\mathfrak{m}_{v}$. If, given any $a \in \mathfrak{m}_{v}$, there is an element $b \in Z_{D}$ such that $1+a=b^{2}$, then $D$ decomposes as a tensor product of $*$-closed quaternion algebras over $Z_{D}$. In particular, this occurs if $v$ restricted to $Z_{D}$ is Henselian.

On the other hand, examples can be constructed $[7, \S 4]$ to show that not all c-valued division rings must decompose as tensor products of quaternion algebras.

Places As usual, valuations have associated places; these are defined in Holland $[20, \S 2]$ for $*$-valuations. A place $\pi$ on a skew field $D$ will be called a $*$-place if it preserves the involution and satisfies $\pi\left(d^{*} d^{-1}\right) \neq \infty$ for all $d \in D^{\times}$. These are studied in some detail in Craven [12], where much of Lam [27, §§9-11] is extended to $*$-places. Just as every ordering of a commutative field has an associated place into the real numbers, $*$-orderings have associated places into the real quaternions $\mathbb{H}$ by the work of Holland mentioned earlier [20, §4]. To obtain uniqueness, one must consider two places equivalent if they differ by an automorphism of $\mathbb{H}$. Let $\mathcal{M}(D)$ denote the set of such equivalence classes of $*$-places. A major result making this set tractable is the following.

Theorem 3.7 Craven [12, Theorem 2.5] Elements of $\mathcal{M}(D)$ are determined by their action on $S(D)$.

The set $\mathcal{M}(D)$ can be given the quotient topology from $X_{D}$, making it a compact Hausdorff space, proved much as in the commutative case. As for commutative fields, we define the real holomorphy ring

$$
\mathcal{H}(D)=\bigcap_{P \in X_{D}} A(P) .
$$

The main results known about $\mathcal{H}(D)$ are that it is a (noncommutative) Prüfer domain (see Craven [12, Corollary 5.2]) and its elements are precisely those $d \in D$ such that there is some positive integer which bounds $d d^{*}$ uniformly with respect to all $*$-orderings $[12$, Theorem 5.3]. 


\section{Spaces of orderings and hermitian forms}

Baer introduced his notion of ordering precisely because of his interest in hermitian forms. His main theorem in [2, Chap. IV, App. I] is a generalization of Sylvester's theorem of inertia. In spite of this, there has been far less work done on this topic than on the valuation theory for orderings. The general definitions of hermitian forms and Witt groups can be found in books such as Scharlau [40], but not the connection with orderings. At this point, we no longer consider c-orderings as their multiplicative properties do not behave well with respect to hermitian forms.

The main efforts to carry over the reduced theory of quadratic forms to *fields, viewing forms as functions on a topological space of orderings, have been by Craven and Smith [11, 15, 16], though an involution was introduced long ago into the commutative theory by Knebusch, Rosenberg and Ware [26]. A hermitian form over $(D, *)$ can be diagonalized to have the form $\phi=\left\langle a_{1}, a_{2}, \ldots, a_{n}\right\rangle$, where $a_{i} \in S(D)^{\times}, i=1, \ldots, n$. We shall denote the Witt group of anisotropic hermitian forms over $(D, *)$ by $W(D, *)$. The basic definitions and properties of hermitian forms over $(D, *)$ and the definition of the Witt group can be found in Scharlau [40, Chapter 7]. Independent of the question of orderings, there has been some work on extending Springer's Theorem for quadratic forms. In [32], Lewis considers the kernel and image of the induced homomorphism $W(F) \rightarrow W(D, *)$, where $D$ is an odd dimensional central simple algebra over a field $F$ and the involution fixes $F$. He shows that the kernel is not zero in general, as it is in the commutative case for odd degree extension fields, and is able to give quite general conditions under which it is zero.

The involution on $D$ works with the Baer orderings and with the hermitian forms in compatible ways, so that one obtains

Theorem 4.1 Craven [11, Theorem 2.5] There exists a bijective correspondence between Baer orderings of $(D, *)$ and group homomorphisms $\sigma: W(D, *) \rightarrow \mathbb{Z}$ satisfying $\sigma(\langle 1\rangle)=1$ and $\sigma(\langle a\rangle)= \pm 1$ for all $a \in S(D)^{\times}$.

The situation for $*$-orderings works particularly well, just as $*$-orderings work well with valuation theory. A canonical factor group of $W(D, *)$, denoted $W R(D, *)$, is defined in [11] on which one can define an associative product $\langle a\rangle \cdot\langle b\rangle=\langle(a b+$ ba) $/ 2\rangle$ making $W R(D, *)$ into a ring. We shall use the same notation for elements of this quotient group as for the hermitian forms which represent the equivalence class. The ring homomorphisms of $W R(D, *)$ into $\mathbb{Z}$ correspond to the $*$-orderings of $(D, *)$, so that there is a "total signature mapping" $W R(D, *) \rightarrow \mathcal{C}\left(X_{D}, \mathbb{Z}\right)$, where $\mathrm{e}\left(X_{D}, \mathbb{Z}\right)$ is the ring of continuous functions from $X_{D}$ to the ring of integers with the discrete topology. The main purpose of the paper [11] is to compute the kernel and image of this mapping (actually in the somewhat more general case of an arbitrary preordering in place of $S(D) \cap \Sigma(D))$.

Theorem 4.2 Craven $\left[11\right.$, Theorem 6.1] The kernel of $W R(D, *) \rightarrow \mathcal{C}\left(X_{D}, \mathbb{Z}\right)$ is additively generated by elements of the form

$$
\left\langle a,-\left(a d+d^{*} a\right)\right\rangle, \quad a \in S(D)^{\times}, d \in \Sigma(D) .
$$

The computation of the image uses the theory of abstract Witt rings and spaces of orderings developed by Marshall [33]. Sections 3, 4, 5, 14, and 15 of Lam [27] are generalized to $*$-fields in order to prove that the image of $W R(D, *)$ is a space of orderings in this abstract sense. Using the abstract theory, one then obtains 
a computation of the image, a direct generalization of the theorem of Becker and Bröcker for formally real fields.

Theorem 4.3 Craven [11, Theorem 6.8] A function $f \in \mathcal{C}\left(X_{D}, \mathbb{Z}\right)$ is in the image of $W R(D, *)$ if and only if for any fan $T$ with $X_{T}$ finite, we have

$$
\sum_{P \in X_{T}} f(P) \equiv 0 \quad\left(\bmod \left|X_{T}\right|\right) .
$$

More recently, Theorem 4.1 has been used as a basis for trying to understand the more general situation of Baer orderings and hermitian forms. Since the abstract structure of the Witt group does not carry much information (unlike the ring structure, when there is one), a different approach has been taken in Craven and Smith [15]. This paper works with the subring $W S(D, *)$ of $\mathcal{C}\left(Y_{D}, \mathbb{Z}\right)$ generated by the image of $W(D, *)$. For fields, $Y_{D}$ is generally much larger than $X_{D}$ and this ring is more complicated, though it is determined by the reduced Witt ring. But when $D$ is noncommutative, it provides a way of obtaining information about the forms even if $D$ has no $*$-orderings, such as when $D$ is finite dimensional over its center. While the rings turn out to be more complicated than Witt rings of fields, they can be discussed either in the context of abstract Witt rings as originally defined by Knebusch, Rosenberg and Ware in the early 1970's, or in terms of prespaces of orderings as recently defined in Andradas, Bröcker and Ruiz [1]. But understanding these rings requires a new operation besides the usual product and group ring constructions.

Theorem 4.4 Craven and Smith [15, Theorem 2.8] Let $(D, *)$ be $a *$-field and assume that $Y_{D}^{v} \neq \emptyset$ is the set of all Baer orderings of $D$ which are compatible with a smooth $*$-valuation $v$. Let $\left(D_{v}, *\right)$ be the residue field with the induced involution, and let $g=\left|S\left(\Gamma_{v}\right) / 2 \Gamma_{v}\right|$, which we assume to be finite. Then

$$
W_{v}(D) \cong\left(\bigotimes_{1}^{g} W S\left(D_{v}, *\right)\right)\left[\mathbb{Z}_{2}^{g-1}\right],
$$

where $W_{v}(D)$ is the quotient ring of $W S(D, *)$ obtained by restricting all functions on $Y_{D}$ to the subset $Y_{D}^{v}$.

Thus we see that instead of the group ring construction for the case of ordinary orderings, there is now also a tensor product involved (over the ring of integers $\mathbb{Z}$ ).

For odd-dimensional $*$-fields, work of Leung [30, Theorem 5.9] reduces the computation of these rings to looking at the field of central symmetric elements. Leung accomplishes this by showing that a Baer ordering $P$ of $D$ consists precisely of those elements whose reduced norm is also in $P$. This gives rise to the following theorem.

Theorem 4.5 Craven and Smith [15, Theorem 4.1] Let $(D, *)$ be a $*$-field with center $Z_{D}$ and let $Z_{0}=Z_{D} \cap S(D)$. Assume that $\left[D: Z_{D}\right]$ is odd. The image of $W(D, *)$ in $\mathcal{C}\left(Y_{D}, \mathbb{Z}\right)$ is the homomorphic image of $W\left(Z_{0}\right)$ under the composition

$$
W\left(Z_{0}\right) \rightarrow W\left(Z_{D}, *\right) \rightarrow W(D, *) \rightarrow \mathcal{C}\left(Y_{D}, \mathbb{Z}\right) .
$$

In particular, the homomorphism $W S\left(Z_{0}\right) \rightarrow W S\left(Z_{D}, *\right) \rightarrow W S(D, *)$ is surjective and is defined by restriction of functions from $Y_{Z_{0}}$ to $Y_{Z_{0}} / T_{0} \cong Y_{D}$; the space $Y_{Z_{0}} / T_{0}$ is the space of all semiorderings of $Z_{0}$ containing the intersection of all the weak $*$-orderings of $D$ intersected with $Z_{0}$. 
Of course, a fundamental issue is how much information is lost in going from $W(D, *)$ to $W S(D, *)$. There is as yet, no good understanding of the kernel. One can, however, decide when the kernel is zero. We shall say that $(D, *)$ is strongly pythagorean if whenever $r \in S(D), d_{i} \in D$, there exists an element $d \in D$ such that $\sum d_{i} r d_{i}^{*}=d r d^{*}$. When $*$ is the identity, this becomes the usual concept of a pythagorean field, as it only says that any sum of squares is again a square.

Theorem 4.6 Craven and Smith [16, Theorem 2.6] Let $(D, *)$ be formally real. The $*$-field $D$ is strongly pythagorean if and only if the total semisignature mapping $W(D, *) \rightarrow \mathcal{C}\left(Y_{D}, \mathbb{Z}\right)$ is one-to-one. If these hold, the Witt group $W(D, *)$ is torsion free.

Strongly pythagorean $*$-fields have particularly nice properties. For them, Baer orderings have something of an Artin-Schreier theory. For example, formal reality is equivalent to the existence of a Baer ordering, the intersection of all Baer orderings is the set of sums of norms, and weakly isotropic hermitian forms are precisely those which are totally indefinite with respect to all Baer orderings.

Finding strongly pythagorean $*$-fields seems to be much more complicated than in the case where $*$ is the identity, in which case one always has a pythagorean closure of any formally real field. The simplest cases to analyze are the commutative *-fields and standard quaternion algebras. For nonstandard quaternion algebras, we have the following.

Theorem 4.7 Craven and Smith [16, Theorem 3.4] Let $F$ be a formally real pythagorean field and let $D=\left(\frac{a, b}{F}\right)$ be a nonstandard quaternion division algebra over $F$ with an involution $*$ of the first kind. As above, we assume that $i^{2}=a, j^{2}=$ $b$, where $i, j \in S(D)$.

1. Assume $F$ has a 2-henselian real valuation $v$ for which $v(a), v(b) \notin 2 \Gamma_{v}$. Then $(D, *)$ is *-pythagorean, but may not be formally real.

2. If $v(a b) \notin 2 \Gamma_{v}$ also holds, then $(D, *)$ is strongly pythagorean and has a Baer ordering.

3. If $(D, *)$ is strongly pythagorean and has a Baer ordering, then $F$ has a nontrivial real valuation $v$ in which $v(a), v(b), v(a b) \notin 2 \Gamma_{v}$. If all orderings of $F$ are compatible with $v$, then $v$ is 2-henselian.

Existence of Baer orderings A very different use of forms also exists. A standard quaternion division algebra $\left(\frac{a, b}{F}\right)$ has a Baer ordering if and only if the form $\langle 1,-a,-b, a b\rangle$ is $T$-anisotropic and $T=\left\{\sum x_{i} x_{i}^{*} \mid x_{i} \in F\right\}$ is a preordering of $F \cap Z(D)$. Leung [31] has extended this to show that there exists a form which determines whether a nonstandard quaternion algebra has a Baer ordering. $\mathrm{He}$ does this also for certain tensor products of quaternion algebras.

\section{Extending the theory to rings}

To apply the ideas here to $\mathrm{C}^{*}$-algebras, one must extend the theory from skew fields to rings with involution. In fact, one would hope that the theory could be extended to arbitrary $*$-rings, giving a noncommutative version of real algebraic geometry. Marshall has recently taken some major strides in this direction (cf. Marshall [34]).

Marshall works primarily with the stronger multiplicative form of orderings. He defines a *-ordering for a ring $A$ (containing 1 and having characteristic zero) 
essentially as in Definition 2.6 plus adding an axiom to handle the intersection $P \cap-P:$

Definition 5.1 A *-ordering on $A$ is a subset $P$ of the symmetric elements $S(A)$ satisfying $1 \in P,-1 \notin P, r P r^{*} \subseteq P$ for all $r \in A, P \cup-P=S(A), a, b \in P$ implies $a b+b a \in P$ and for any $a, b \in S(A)$,

$$
a b a \in P \cap-P \Longrightarrow a \in P \cap-P \text { or } b \in P \cap-P \text {. }
$$

This final condition makes the support of $P$ extend to a prime ideal of $A$ and allows a real spectrum to be defined. Factoring out the support of $P$ allows Marshall to deal with domains and use valuation theory in a manner similar to that for *orderings of skew fields, even though these domains may not have skew fields of fractions. After much work, he is able to generalize the theorem, mentioned in Section 4 , that the image of $W R(D, *)$ is a space of orderings:

Theorem 5.2 Let $\operatorname{Sper}_{*}(A)$ be the space of all *-orderings of a*-ring $A$, and let $G_{*}(A)$ be the set of functions $\bar{a}: \operatorname{Sper}_{*}(A) \rightarrow\{-1,0,1\}$ induced by symmetric elements $a \in A$. Then $\left(\operatorname{Sper}_{*}(A), G_{*}(A)\right)$ is a space of signs (cf. [1, Chapter 3]).

A space of signs is also called an abstract real spectrum and is the natural generalization to commutative rings of a space of orderings. For a $C^{*}$-algebra, the space of signs is shown to be real closed (in the usual sense of spectral spaces).

Craven and Smith [17] have taken the first steps toward extending the work of Craven [14] to $*$-rings. They analyze how a given $*$-ordering $P$, as a subset of the symmetric elements, can extend to a multiplicatively closed ordering on a larger set of elements. A complete answer is given for Ore $*$-domains by making use of the field of fractions, though the details are surprisingly complicated. Only a partial answer is given for a domain which is not Ore. An example is provided to show that the problem is not vacuous: the free algebra on two indeterminates over the ring of integers (with the involution which switches the indeterminates) has a $*$-ordering $P$ with $P \cap-P=\{0\}$.

\section{References}

1. C. Andradas, L. Bröcker and J. Ruiz [1996] Constructible sets in real geometry, Springer Verlag, Berlin.

2. R. Baer [1952] Linear algebra and projective geometry, Academic Press, New York.

3. M. Chacron [1982] c-orderable division rings with involution, J. Algebra 75, 495-522.

4. M. Chacron [1982] Representation of partial *-orderings, Comm. Algebra 10, 581-609.

5. M. Chacron [1989] c-valuations and normal c-orderings, Canad. J. Math. 41, 14-67.

6. M. Chacron [1990] A symmetric version of the notion of c-ordering, Comm. Algebra 18, 3059-3084.

7. M. Chacron and A. Wadsworth [1990] On decomposing c-valued division rings, J. Algebra 134, 182-208.

8. T. Craven [1989] Orderings and valuations on *-fields, Rocky Mountain J. Math. 19,629-646.

9. T. Craven [1988] Approximation properties for orderings on $*$-fields, Trans. Amer. Math. Soc. 310, 837-850.

10. T. Craven [1990] Characterization of fans in *-fields, J. Pure Appl. Algebra 65, 15-24.

11. T. Craven [1992] Witt groups of hermitian forms over $*$-fields, J. of Algebra 147, 96-127.

12. T. Craven [1990] Places on *-fields and the real holomorphy ring, Comm. Algebra 18, 27912820.

13. T. Craven [1995] Orderings, valuations and hermitian forms over *-fields, Proc. Sympos. Pure Math. 58, 149-160.

14. T. Craven [1996] Extension of orderings on $*$-fields, Proc. Amer. Math. Soc. 124, 397-405. 
15. T. Craven and T. Smith [1999] Hermitian forms over ordered $*$-fields, J. Algebra 216, 86-104.

16. T. Craven and T. Smith [2000] Pythagorean *-fields, J. Algebra 225, 487-500.

17. T. Craven and T. Smith Ordered $*$-rings, J. Algebra (to appear).

18. D. Handelman [1981] Rings with involution as partially ordered abelian groups, Rocky Mountain J. Math. 11, 377-381.

19. S. Holland, Jr. [1977] Orderings and square roots in *-fields, J. Algebra 46, 207-219.

20. S. Holland, Jr. [1980] *-valuations and ordered $*$-fields, Trans. Amer. Math. Soc. 262, 219-243.

21. S. Holland, Jr. [1981] Erratum to: "*-valuations and ordered *-fields", Trans. Amer. Math. Soc. 267, 333.

22. S. Holland, Jr. [1986] Strong orderings of $*$-fields, J. Algebra 101, 16-46.

23. S. Holland, Jr. [1987] Baer ordered *-fields of the first kind, Israel J. Math. 57, 365-374.

24. I. Idris [1986] *-valuated division rings, orderings and elliptic hermitian spaces, Ph.D. thesis, Carleton University, Ottawa, Canada.

25. I. Idris [1989] Jordan ordering of a division ring with involution, Arabian J. Sci. Engrg. 14, $527-535$.

26. M. Knebusch, A Rosenberg and R. Ware [1973] Signatures on semilocal rings, J. Algebra 26, $208-250$.

27. T. Y. Lam [1983] Orderings, valuations and quadratic forms, Conference Board of the Mathematical Sciences No. 52, Amer. Math. Soc., Providence, RI.

28. K. H. Leung [1993] Weak *-orderings on $*$-fields, J. Algebra 156, 157-177.

29. K. H. Leung [1994] Strong approximation property for Baer orderings on *-fields, J. Algebra 165, 1-22.

30. K. H. Leung [1995] Weakly *-ordered *-fields, J. Pure Appl. Algebra 99, 221-238.

31. K. H. Leung [2000] Forms and Baer ordered $*$-fields, Israel J. Math. 116, 1-19.

32. D. W. Lewis [1989] Hermitian forms over odd dimensional algebras, Proc. Edinburgh Math. Soc. 32, 139-145.

33. M. Marshall [1980] Abstract Witt rings, Queen's Papers in Pure and Appl. Math, No. 57, Queen's University, Kingston, ON.

34. M. Marshall [2000] *-orderings on a ring with involution, Comm. Algebra 28, 1157-1173.

35. M. McDonough [1997] Witt rings of $*$-fields, Ph.D. thesis, University of Cincinnati, Cincinnati, Ohio.

36. P. Morandi and B. Sethuramen [1994] Indecomposable division algebras with a Baer ordering, Comm. Algebra 22, 5401-5418.

37. P. Morandi and B. Sethuramen [1995] Noncrossed product division algebras with a Baer ordering, Proc. Amer. Math. Soc. 123, 1995-2003.

38. P. Morandi and A. Wadsworth [1989] Baer orderings with noninvariant valuation ring, Israel J. Math. 68, 241-255.

39. A. Prestel [1984] Lectures on formally real fields, Springer Lecture Notes No. 1093, SpringerVerlag, Berlin.

40. W. Scharlau [1985] Quadratic and hermitian forms, Springer-Verlag, Berlin.

41. O. Schilling [1950] The theory of valuations, Math. Surveys No. 4, Amer. Math. Soc., Providence, RI. 